\title{
RECENT RESULTS ON DIFFRACTION AT HERA
}

\author{
Sergey Levonian ${ }^{a}$ \\ DESY, Notkestraße 85, 22607 Hamburg, Germany
}

\begin{abstract}
Four new measurements are presented from the area of diffractive and exclusive production at HERA. Isolated photons are studied in diffractive photoproduction, while open charm cross section and cross-section ratio $\sigma_{\psi(2 S)} / \sigma_{J / \psi(1 S)}$ are measured in diffractive deep-inelastic scattering (DIS) regime. Finally, exclusive $\rho^{0}$ meson photoproduction associated with a leading neutron is investigated for the first time at collider experiments.
\end{abstract}

\section{Introduction}

Diffraction is an important and challenging part of physics landscape at the electron-proton collider HERA. It represents a complicated interplay of soft and hard phenomena, thus providing rich opportunities for QCD studies. Diffractive $e p$ scattering at high photon virtualities $Q^{2}$ for the first time allows a partonic content of the Pomeron, a central object in diffractive physics deeply related to QCD vacuum, to be probed. Using these diffractive parton densities (DPDF) to predict various final states in $e p$ and also in $p p$ diffraction relies on collinear factorisation theorem [1] and hence provides crucial tests of major concepts and overall consistency of QCD picture of diffraction in high energy particle collisions.

Below, four new measurements are presented, performed by the H1 and ZEUS collaborations, which benefit from large integrated luminosity, or from the detector upgrades implemented for HERA2 running period (2004-2007).

\section{Isolated photons in diffractive photoproduction}

Prompt photons in diffractive events are interesting in two respects. First, they are direct messengers from hard subprocess which, unlike jets, are not affected by fragmentation. In addition, photons couple to charged partons only and hence they probe quark content of the Pomeron in diffractive processes. ZEUS Collaboration studied this process [2] using two data samples of $91 \mathrm{pb}^{-1}$ (HERA1) and $374 \mathrm{pb}^{-1}$ (HERA2) respectively. High transverse energy photons $4<E_{T}^{\gamma}<15 \mathrm{GeV}$ are selected in central pseudo-rapidity range $-0.7<\eta^{\gamma}<0.9$ in diffractive photoproduction: $Q^{2}<1 \mathrm{GeV}^{2}, 0.2<y<0.7, x_{\mathbb{P}}<0.03$, where $x_{\mathbb{P}}$ is the fraction of the proton energy taken by the Pomeron. In $\sim 85 \%$ of such events an isolated photon is accompanied by a jet with $E_{T}^{j e t}>4 \mathrm{GeV}$. A template fit of the energy weighted cluster width is employed to statistically separate prompt photons and background originating mainly from $\pi^{0}$ and $\eta$ decays (see Fig. 1a). The data are corrected to the hadron level and compared

\footnotetext{
${ }^{a}$ E-mail: levonian@mail.desy.de
} 
with theory as provided by the Rapgap MC program [3], normalised to the data. In Fig. 1b the cross section is given as a function of $z_{\mathbb{P}}$, a fraction of the Pomeron momentum transferred to the hard subprocess. The data show prominent peak at largest $z_{\mathbb{P}}$ which is not expected and not reproduced by the Rapgap, containing so called resolved Pomeron contributions only. All other distributions are well described by this model. These events at $z_{\mathbb{P}} \approx 1$ are interpreted as a first evidence of direct Pomeron contribution.
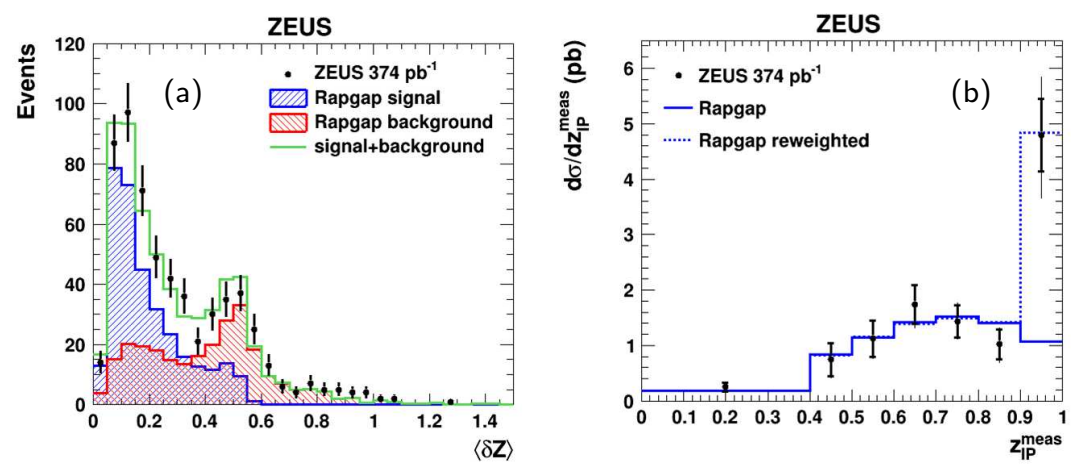

Figure 1: (a) The distribution of the energy-weighted cluster width for isolated photon candidates. (b) Differential cross section as a function of $z_{\mathbb{P}}$ for diffractive events containing an isolated photon and a jet, compared to normalised prediction from Rapgap MC.

\section{$3 \quad D^{*}$ in diffractive DIS}

Contrary to prompt photons, charm in diffractive $e p$ scattering is produced predominantly via boson-gluon fusion, and thus is strongly sensitive to the gluon content of the Pomeron. $D^{*}$ mesons reconstructed via so called 'golden' decay mode $D^{*+} \rightarrow D^{0} \pi_{\text {slow }}^{+} \rightarrow\left(K^{-} \pi^{+}\right) \pi_{\text {slow }}^{+}+C$.C. provide a convenient way of tagging open charm production. H1 Collaboration has used $281 \mathrm{pb}^{-1}$ data sample to measure $D^{*}$ cross section in diffractive DIS [4] for $p_{T, D^{*}}<1.5 \mathrm{GeV}$ and $-1.5<\eta_{D^{*}}<1.5$ in the range of $5<Q^{2}<100 \mathrm{GeV}^{2}$ and $0.02<y<$ 0.65. Diffractive events are selected using Large Rapidity Gap signature and correspond to the following phase space: $x_{\mathbb{P}}<0.03,|t|<1 \mathrm{GeV}^{2}$ and proton dissociative mass $M_{Y}<1.6 \mathrm{GeV}$.

In Fig. 2 the measured differential cross sections are confronted with the NLO QCD calculations [5] which use $\mu_{r}^{2}=\mu_{f}^{2}=m_{c}^{2}+Q^{2}$ scale choice and DPDF set [6] as determined in $\mathrm{H} 1$ inclusive diffraction. For $c \rightarrow D^{*}$ fragmentation the Kartvelishvili parametrisation is used with the parameters fixed by the H1 inclusive $D^{*}$ measurements. NLO QCD describes the data fairly well both in shapes and in absolute normalisation. This supports the universality of charm 

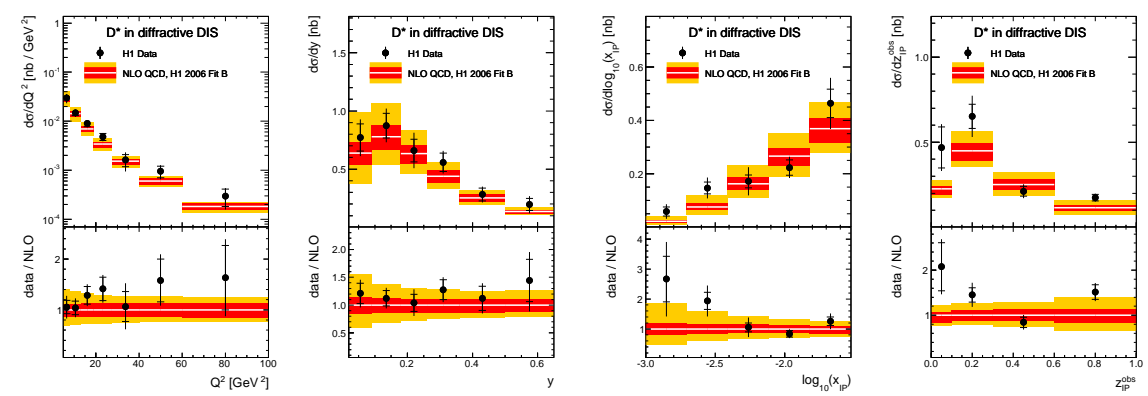

Figure 2: $D^{*}$ meson cross sections as a function of the $Q^{2}$, the inelasticity $y$, and the diffractive variables $x_{\mathbb{P}}$ and $z_{\mathbb{P}}^{\text {obs }}$ as compared to NLO QCD prediction.

fragmentation and the validity of QCD factorisation ansatz in diffractive DIS.

\section{Ratio $\sigma_{\psi(2 S)} / \sigma_{J / \psi(1 S)}$ in exclusive DIS}

An important testing ground for theoretical concepts and quantitative calculations in diffraction is provided by the study of exclusive vector meson production at HERA $e+p \rightarrow e+V+Y$. The exclusive deep inelastic electroproduction of $\psi(2 S)$ and $J / \psi(1 S)$ has been studied with the ZEUS detector in the kinematic range $2<Q^{2}<80 \mathrm{GeV}^{2}, 30<W<210 \mathrm{GeV}$ and $|t|<1 \mathrm{GeV}^{2}$ [7]. Since these two charmonium states have same quark content, but different radial distributions of the wave functions (w.f.), their cross section ratio allows QCD predictions of the w.f. dependence on the $\bar{c} c$-proton cross section to be tested. In particular, a suppression of $\psi(2 S)$ relative to $J / \psi(1 S)$ is expected due to the node in the $\psi(2 S)$ w.f. leading to the destructive interference of the contributions from small and large $\bar{c} c$ dipoles to the production amplitude.

Since the reactions studied are statistically limited all available data have been used, amounting to $468 \mathrm{pb}^{-1}$. The final sample contains $\sim 2500 \mathrm{~J} / \psi(1 S)$ and $\sim 190 \psi(2 S)$ events. After correcting for the detector acceptance, efficiency and the branching ratios, the cross section ratio $R=\sigma_{\psi(2 S)} / \sigma_{J / \psi(1 S)}$ was determined in bins of $Q^{2}, W$ and $|t|$ with statistical precision of $\sim 20 \%$ and systematic uncertainty $\sim 10 \%$. While as a function of $W$ and $|t|$ the values of $R$ are found to be compatible with a constant, the $Q^{2}$ dependence shows a positive slope with the significance of $\sim 2.5 \sigma$.

In Fig. 3 the results are compared to the previous $\mathrm{H} 1$ measurements [8] and confronted with a set of QCD models. One can see, that all models correctly predict the suppression strength at $Q^{2}=0$ and qualitatively reproduce the rise of $R$ with $Q^{2}$, however with a large spread in its magnitude. Thus, the experimental data, albeit with relatively large uncertainties, show a definite discriminating power allowing to disfavour some extreme model predictions. 

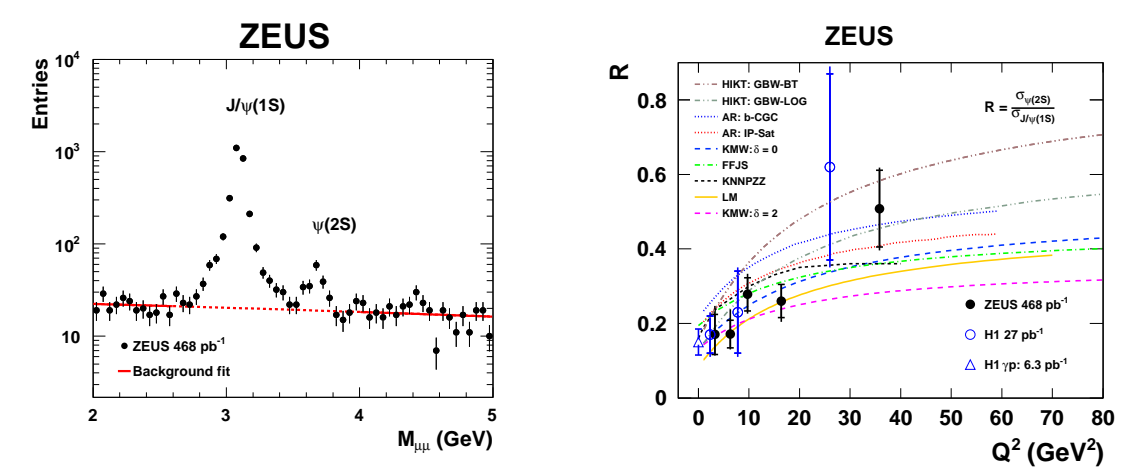

Figure 3: Two-muon mass distribution, $M_{\mu \mu}$, for exclusive dimuon events (left panel) and the cross-section ratio $R=\sigma_{\psi(2 S)} / \sigma_{J / \psi(1 S)}$ as a function of $Q^{2}$ (right panel). HERA measurements are compared to different QCD model predictions (see ref. in [7]).

\section{$5 \quad$ Exclusive $\rho^{0}$ photoproduction with a leading neutron}

Measurements of leading baryon production in high energy particle collisions provide important inputs for the theoretical understanding of strong interactions in the soft, non-perturbative regime. The aim of the analysis presented here is to investigate exclusive $\rho^{0}$ production on virtual pions in the photoproduction regime at HERA and to extract for the first time experimentally the quasi-elastic $\gamma \pi \rightarrow \rho^{0} \pi$ cross section. At small transverse momenta squared $t \rightarrow 0$ dominant contribution comes from one-pion-exchange (OPE) diagram shown in Fig. 4a.

The analysis is based on $\sim 6600$ events, containing only two charged pions from $\rho^{0}$ decay and a leading neutron with energy $E_{n}>120 \mathrm{GeV}$, and nothing else above noise level in the detector. This ensures the exclusivity and limits the dissociative background to the range $M_{Y}<1.6 \mathrm{GeV}$. The sample corresponds to an integrated luminosity of $1.16 \mathrm{pb}^{-1}$, collected by a special minimum bias track trigger in the years 2006-2007 at $\sqrt{s_{e p}}=319 \mathrm{GeV}$. Diffractive dissociation background is modelled by the Diffvm program [10] and statistically separated from the signal, $\gamma p \rightarrow \rho^{0} n \pi^{+}$, on the basis of the neutron energy distribution shape. It amounts to $(34 \pm 5) \%$ and gives the dominant experimental uncertainty to the cross section measurement. Further details of the analysis can be found in [9].

The cross sections for the reaction $\gamma p \rightarrow \rho^{0} n \pi^{+}$are measured for two ranges of the neutron transverse momentum, $p_{T, n}: p_{T, n}<0.69 E_{n}$, corresponding to the full angular acceptance of the neutron calorimeter, $\theta_{n}<0.75 \mathrm{mrad}$, and for so called OPE safe range, $p_{T, n}<0.2 \mathrm{GeV}$. In Fig. $4 \mathrm{~b}$ the data are compared to the predictions, based on different models for the pion flux. The shape of $x_{L}$ 
(a)

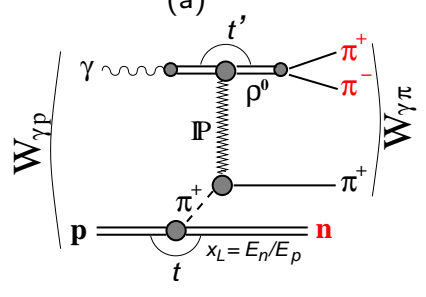

(b)

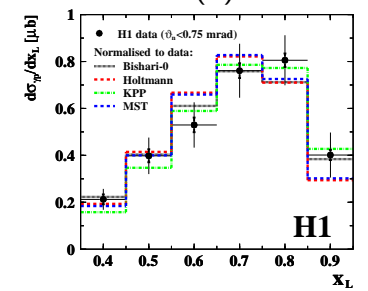

(c)

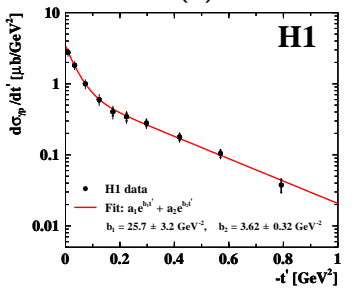

Figure 4: (a) OPE diagram for exclusive photoproduction of $\rho^{0}$ mesons associated with leading neutrons at HERA. (b) Differential cross section $\mathrm{d} \sigma_{\gamma p} / \mathrm{d} x_{L}$ in the range $20<W_{\gamma p}<$ $100 \mathrm{GeV}$ compared to the predictions based on different versions of pion fluxes (see ref. in [9]). (c) Differential cross section $\mathrm{d} \sigma_{\gamma p} / \mathrm{d} t^{\prime}$ of $\rho^{0}$ mesons fitted with the sum of two exponential functions. The inner error bars in $(b, c)$ represent statistical and uncorrelated systematic uncertainties added in quadrature and the outer error bars are total uncertainties, excluding an overall normalisation error of $4.4 \%$.

distribution is described by most of the models, although some of them can be ruled out already at that level [9]. The pion flux models compatible with the data in shape of the $x_{L}$ distribution are used to extract the photon-pion cross section at $\left\langle W_{\gamma \pi}\right\rangle=24 \mathrm{GeV}$ from $\mathrm{d} \sigma / \mathrm{d} x_{L}$ in the OPE approximation:

$$
\sigma\left(\gamma \pi^{+} \rightarrow \rho^{0} \pi^{+}\right)=2.33 \pm 0.34(\exp )_{-0.40}^{+0.47}(\text { model }) \mu \mathrm{b},
$$

where the experimental uncertainty includes statistical, systematic and normalisation errors added in quadrature, while the model error is due to the uncertainty in the pion flux integral obtained for the different flux parametrisations compatible with the data. Taking a value of $\sigma\left(\gamma p \rightarrow \rho^{0} p\right)=(9.5 \pm 0.5) \mu \mathrm{b}$ at the corresponding energy $\langle W\rangle=24 \mathrm{GeV}$, which is an interpolation between fixed target and HERA measurements, one obtains for the ratio $r_{\mathrm{el}}=$ $\sigma_{\mathrm{el}}^{\gamma \pi} / \sigma_{\mathrm{el}}^{\gamma p}=0.25 \pm 0.06$. This ratio is significantly smaller than the expected value $r_{\mathrm{el}}=\left(\frac{b_{\gamma p}}{b_{\gamma \pi}}\right) \cdot\left(\sigma_{\text {tot }}^{\gamma \pi} / \sigma_{\text {tot }}^{\gamma p}\right)^{2}=0.57 \pm 0.03$ which can be deduced by combining the optical theorem, the eikonal approach relating cross sections with elastic slope parameters and the data on $p p, \pi^{+} p$ and $\gamma p$ elastic scattering. Such a suppression of the cross section is usually attributed to rescattering, or absorptive corrections, which are essential for leading neutron production. For the exclusive reaction $\gamma p \rightarrow \rho^{0} n \pi^{+}$studied here this would imply an absorption factor of $K_{a b s}=0.44 \pm 0.11$.

Finally, the cross section as a function of the four-momentum transfer squared of the $\rho^{0}$ meson, $t^{\prime}$, is presented in Fig. 4c. It exhibits the very pronounced feature of a strongly changing slope between the low- $t^{\prime}$ and the high- $t^{\prime}$ regions, which is characteristic to double peripheral exclusive reactions. In a geometric picture, the large value of slope $b_{1}$ suggests that for a significant part of the data $\rho^{0}$ mesons are produced at large impact parameter values of order $\left\langle r^{2}\right\rangle=2 b_{1} \cdot(\hbar c)^{2} \simeq 2 \mathrm{fm}^{2} \approx\left(1.6 R_{\mathrm{p}}\right)^{2}$. 


\section{Summary}

Four new measurements are presented in the area of diffractive and exclusive channels, by the H1 and ZEUS collaborations, making use of full HERA data samples. Whenever hard scale is present, perturbative QCD calculations are successful. The data show sensitivity to some QCD models parameters. They can also be used to further constrain DPDFs, especially at high $z_{\mathbb{P}}$. Photonpion elastic cross section is extracted for the first time experimentally in the one-pion-exchange approximation. Strong absorptive effects are confirmed in leading neutron production. Since the nature of those is non-perturbative, new experimental results are essential for the tuning of 'Survival Gap Probability' models.

[1] J.C. Collins, Phys. Rev. D57 (1998) 3051. Erratum-ibid. 61, 019902 (2000).

[2] H. Abramowicz et al. [ZEUS Collab.], Phys. Rev. D96 (2017) 032006.

[3] H. Jung, Comp. Phys. Commun. 86 (1995) 147.

[4] V. Andreev et al. [H1 Collab.], Eur. Phys. J. C77 (2017) 340.

[5] B.W. Harris and J. Smith, Phys. Rev. D57 (1998) 2806.

[6] A. Aktas et al. [H1 Collab.], Eur. Phys. J. C48 (2006) 715.

[7] H. Abramowicz et al. [ZEUS Collab.], Nucl. Phys. B909 (2016) 934.

[8] C. Adloff et al. [H1 Collab.], Phys. Lett. B421 (1998) 385; C. Adloff et al. [H1 Collab.], Eur. Phys. J. C10 (1999) 373.

[9] V. Andreev et al. [H1 Collab.], Eur. Phys. J. C76 (2016) 41.

[10] B. List and A. Mastroberardino, Proc. of the Workshop on Monte Carlo Generators for HERA Physics, eds. A.T. Doyle et al., DESY-PROC1999-02 (1999) 396. 